\title{
Study on the forming and sensing properties of laser-sintered TPU/CNT composites for plantar pressure sensors
}

\author{
Yu Zhuang ${ }^{1} \cdot$ Yanling Guo ${ }^{1} \cdot \operatorname{Jian} \mathrm{Li}^{1} \cdot$ Yueqiang $\mathrm{Yu}^{2} \cdot$ Kaiyi Jiang ${ }^{3} \cdot$ Hui Zhang ${ }^{1} \cdot$ Shuai Guo ${ }^{1}$
}

Received: 12 September 2020 / Accepted: 21 December 2020 / Published online: 6 January 2021

(C) The Author(s) 2021

\begin{abstract}
Conductive polymer composites (CPCs) combining with specific microstructures (micropores, microcracks, etc.) can exhibit unique resistance response changes, which can be widely regarded as an effective way to improve sensing performance. This study takes advantage of the characteristics of the formation of tiny pores between crystal grains during selective laser sintering (SLS) processing to introduce a microporous structure into the thermoplastic polyurethane (TPU)/carbon nanotube (CNT) sensing element to prepare a three-dimensional porous conductive structure. The effect of the SLS process on sensing sensitivity, accuracy, and density was studied, and its sensing and forming mechanism were discussed. By adjusting SLS process parameters to control the performance of porous structure sensor elements, a final TPU/CNT sensor element with a wide pressure detection range, high sensitivity, a fast response time, and good stability and durability was developed. Finally, the optimal performance of the developed flexible pressure sensor was successfully used to detect the pressure distribution of the human foot. This study provided a simple and effective research method to develop high-performance flexible pressure sensors.
\end{abstract}

Keywords Selective laser sintering $\cdot$ Pressure-sensing element $\cdot$ Thermoplastic polyurethane $\cdot$ Carbon nanotubes $\cdot$ Conductive polymer composites

\section{Introduction}

Recently, novel resistive-type conductive polymer composite (CPC)-based strain sensors have attracted attention based on their merits of light weight, flexibility, stretchability, and easy processing, thus showing great potential applications in the fields of software robots [1], electronic skins [2], smart wearable devices [3], human movement detection [2, 4, 5], etc. Owing to the flexibility and stretchability of the polymer matrix, CPCs also have great potentials for the detection of various external stimuli (tensile, compression, organic vapor, temperature, etc.) based on the reconstruction of conductive

Yanling Guo

nefugylzy@163.com

1 School of Mechatronics Engineering, Northeast Forestry University, Harbin 150040, China

2 College of Mechanical Science and Engineering, Northeast Petroleum University, Daqing 163318, China

3 School of Engineering and Technology, Northeast Forestry University, Harbin 150040, China networks, which induced significant resistance variation [6-8]. Generally, CPCs are fabricated through combining conductive fillers (Ag nanowire, carbon nanotube [9], graphene [10], etc.) and insulating polymers with proper processing technology (melt mixing, solution mixing, dip-coating, spray coating, etc.).

Carbon nanotubes (CNTs) are often used as conductive filler materials and are good materials for preparing CPCbased strain sensors because of their large specific surface area, aspect ratio, high specific strength and modulus, and hardness and toughness [11, 12]. Michelis et al. [13] built a highly reproducible and hysteresis-free flexible strain sensor using inkjet printing CNTs on ethylene tetrafluoroethylene sheets. Liu et al. [14] prepared a lightweight porous thermoplastic polyurethane (TPU)/CNT sensor using the thermally induced phase separation (TIPS) technology, which has ultrahigh compressibility and fast sensing capacity over a wide strain range (up to 90\%). Lee et al. [15] applied silicone rubber to polyurethane foam and further dip-coated it with multiwalled carbon nanotube (MWCNT) dispersed TPU ink to produce a sensor that has good repeatability, high durability, and fast response speed, and the sensor can measure pressures of less than $100 \mathrm{~Pa}$ and more than $200 \mathrm{kPa}$. However, the 
surfaces of these CPC-based strain sensors are generally planar, as they are formed by hot compression and casting in a specific mold, which means that these pressure sensors cannot completely fit objects with complex curved surfaces, resulting in measurement errors.

Additive manufacturing (AM) is currently the fastestgrowing manufacturing method. It allows the manufacturing of products with complex geometry and high dimensional accuracy, as well as high strength [16]. Following the increasing interest in $\mathrm{AM}$, some AM technologies such as stereolithography, fused filament fabrication, and fused deposition modeling technologies are gradually popularized. Nevertheless, this approach has a limited range of applications due to the relatively low mechanical strength of applied polymer materials and several technological restrictions [17-20]. Recent development of AM methods, i.e., selective laser melting, electron beam melting, selective laser sintering (SLS), and laser engineering net shaping (LENS), offers new possibilities to conduct investigations on complex surfaces and lattice structures. Selective laser melting and LENS technologies use a high-power fiber laser with a wavelength of $1.064 \mu \mathrm{m}$ as the energy source; these technologies are more suitable for the absorption and melting of metal alloy powders. Selective laser sintering uses a $\mathrm{CO}_{2}$ laser with a wavelength of $10.64 \mu \mathrm{m}$, which is more suitable for sintering thermoplastic polymers, coated sand, and ceramic powder. Additionally, SLS is a wide range of processing materials and no support material is needed $[21,22]$, which make it more suitable for preparing flexible pressure-sensing elements with a complex curved surface structure. Among the commonly used polymer materials for laser sintering, TPU is an excellent electrical insulator that exhibits high elasticity, flexibility, and environmental stability. It is widely used in textile, medical, automotive, aerospace, aviation, and other fields, and it is an ideal base material for developing CPCs. Additionally, due to the unique forming process mechanism of laser sintering, it is possible to produce inherently porous objects. These pressure-sensing elements with a porous structure can greatly improve the sensitivity and gauge coefficient of the sensing element [23].

Laser sintering is a very complex thermophysical process, which depends not only on the accuracy of the SLS equipment and the thermophysical parameters of the material but also on the process parameters of the SLS [24, 25]. For commercial SLS equipment, only a few process parameters, such as laser power $(P)$, layer thickness $(h)$, scanning speed $(v)$, and scan spacing $(b)$, are relatively easy to control and adjust. The three parameters $(P, v$, and $b)$ need to cooperate to achieve the energy $(E=P /(v \cdot b))$ required for powder sintering. Additionally, the sensitivity of the pressure sensor element studied in this paper is affected not only by the material but also by the porosity of the sensor element. The scan spacing affects the density in the horizontal direction ( $X Y$ plane), and the layer thickness affects the density in building direction $(Z)$.
Therefore, this study uses laser power, layer thickness, and scan spacing as input parameters for experiments. In the research of laser processing and molding optimization, many scholars adopted different experimental methods. Flores et al. [26] used the topology optimization method to optimize the parameters of the lattice structure of the parts, which reduced the manufacturing cost by $53.7 \%$ and the production time by $54.3 \%$ and increased the output. Arisoy et al. [27] used the surface response regression method to study the influence of the cooling rate and thermal gradient during laser processing of nickel alloy (IN625) material, which improved production efficiency.

In this study, TPU was used as the flexible polymer matrix material and CNTs as the conductive filler to print CPC-based pressure-sensing elements using SLS. First, CNT powder was dispersed in TPU powder using a strong acid oxidation method combined with a ball mill dispersion method, and the dispersion of the CNT powder was observed by combining with scanning electronic microscopy (SEM). A three-factor fourlevel orthogonal experimental design was performed, and the influence of the SLS process on the molding quality and sensing performance of the TPU/CNT sensing element was discussed. The optimized TPU/CNT sensing element was tested for its sensing performance (such as piezoresistive sensitivity, pressure measurement range, response time, and stability under cyclic load).

\section{Materials and methods}

\subsection{SLS experimental equipment}

Fabrication of the TPU/CNT sensing element and molding processing were mainly achieved using SLS equipment (AFS-360, Beijing Long Yuan Technology Ltd.) equipped with a $\mathrm{CO}_{2}$ laser generator (laser power of $55 \mathrm{~W}$ and laser spot diameter of $0.20 \mathrm{~mm}$ ). The dimensions of the forming box were $360 \mathrm{~mm} \times 360 \mathrm{~mm} \times 500 \mathrm{~mm}$, with a layer thickness range of 0.1 to $0.3 \mathrm{~mm}$.

\subsection{Materials}

Carbon nanotube powder with an average diameter of 5-15 $\mathrm{nm}$ and an average length of $0.5-1.5 \mu \mathrm{m}$ was supplied by Shenzhen Turing Evolution Technology Co., Ltd., China. Thermoplastic polyurethane powder with an average grain diameter of $80 \mu \mathrm{m}$ (LUVOSINT X92A-1) was purchased from Luvocom, Germany, as shown in Fig. 1a. Concentrated sulfuric acid $\left(\mathrm{H}_{2} \mathrm{SO}_{4}, 96-98 \%\right)$ and concentrated nitric acid $\left(\mathrm{HNO}_{3}, 68 \%\right)$ were supplied by Sinopharm Chemical Reagent Co., Ltd., China. 
Fig. 1 a Micromorphology of the TPU powder. $\mathbf{b}$

Micromorphology of the TPU/ CNT powder
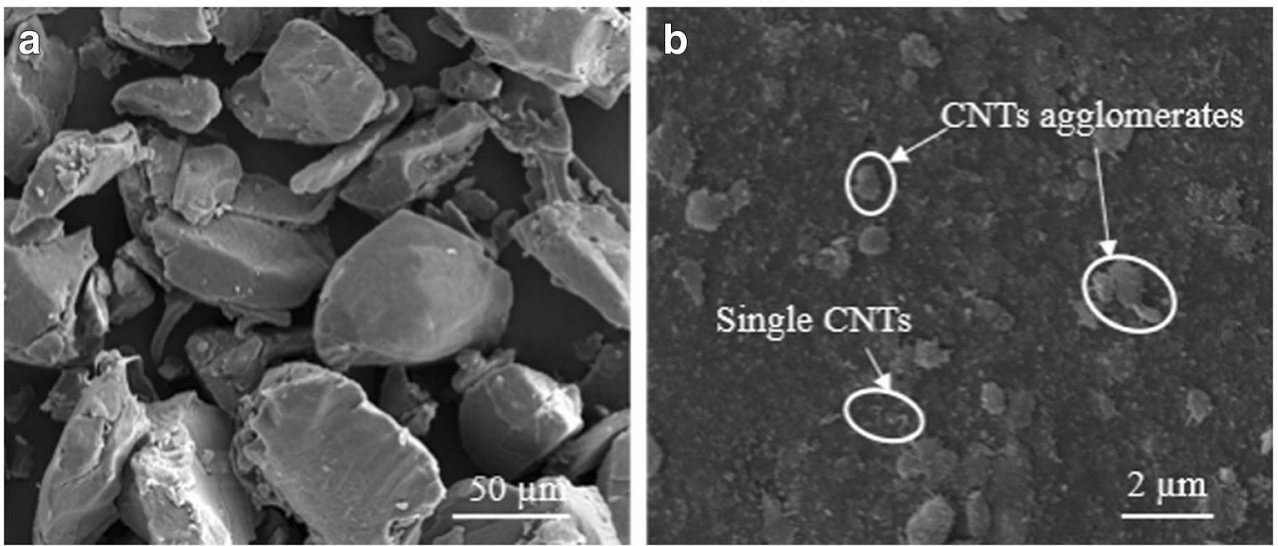

\subsection{Preparation of TPU/CNT composites}

The properties of the TPU/CNT sensing elements depend on the dispersion of the CNTs in the TPU matrix and on the interfacial binding force between the CNTs and TPU. Therefore, they were pre-treated using a strong acid oxidation method to directly modify the surface of the CNTs and improve their dispersion in the TPU matrix [28].

The TPU/CNT composites were prepared as follows: mix concentrated $\mathrm{H}_{2} \mathrm{SO}_{4}$ and $\mathrm{HNO}_{3}$ in a beaker at a ratio of 3:1 with CNTs and magnetically stir them at $80{ }^{\circ} \mathrm{C}$ for $2 \mathrm{~h}$. After standing and stratification, the CNTs were filtered out and washed with deionized water until the $\mathrm{pH}$ was neutral. Then, the CNTs were dried and put into a planetary ball mill with a grinding speed of $240 \mathrm{r} / \mathrm{min}$ and a grinding time of $2 \mathrm{~h}$. The crushed CNT powder and TPU were put into an SHR50A high-speed mixer at a ratio of $0.25 \mathrm{wt} \%$ and stirred for $2 \mathrm{~min}$ at a speed of $700 \mathrm{r} / \mathrm{min}$ to obtain TPU/CNT composite powder. As shown in Fig. 1b, the CNTs are still slightly aggregated after strong acid oxidation and crushing, but they are evenly distributed on the surface of the TPU.

\subsection{Methods}

\subsubsection{Orthogonal experiment design}

The piezoresistive sensitivity, density, and dimensional precision of TPU/CNT sensing elements are important factors to evaluate sensing performance and formability. These factors mainly depend on main SLS process parameters such as laser power, layer thickness, and scan spacing. To determine the range of processing parameters, we conducted some experiments in advance. The experiments showed that both large layer thickness $(0.3 \mathrm{~mm})$ and low laser power $(17 \mathrm{~W})$ led to delamination of the part (Fig. 2a), and no resistance change can be detected. Furthermore, due to the limitation of the SLS equipment's accuracy, the minimum layer thickness was specified as $0.1 \mathrm{~mm}$. High laser power $(30 \mathrm{~W})$ caused excessive sintering, resulting in warping and degrading of parts (Fig. 2b).
Hence, the laser power was set at a range of $19-28 \mathrm{~W}$ and the layer thickness range was $0.1-0.25 \mathrm{~mm}$. Additionally, since the laser is a Gaussian heat source, the scan spacing has a great influence on the heat absorption of the material. When the scan spacing is large, a continuous sintered surface cannot be formed between the scanning lines. If the scan spacing is too small, the sintered powder will absorb too much heat, and the scanning lines will overlap considerably, resulting in excessive sintering. The sintering efficiency and quality will be unsatisfactory, so the scan spacing was set at a range of 0.1-0.25 mm. An SLS experiment was performed using an orthogonal experiment design scheme of three factors and four levels. The test scheme is shown in Table 1.

\subsubsection{Scanning electronic microscopy}

Scanning electronic microscopy (FEI Quanta 200 microscope, Hewlett-Packard Company, Netherlands) was used to observe the morphologies of the TPU/CNT powders and TPU/CNT sensing elements. Since the materials are non-conductive, the specimens were first sputtered with gold using SEM specimen-coating equipment.

\subsubsection{Piezoresistive response tests}

The piezoresistive response experiment equipment was mainly composed of two parts: a mechanical testing machine and a high-precision multimeter, as shown in Fig. 3. The electronic universal testing machine (Byes-3003, Bangyi Precision Meter Co., Ltd.) was used to provide external pressure, and the high-precision multimeter (Victor 8165A) was used to record the resistance change of the TPU/CNT sensing elements under pressure. The upper and lower sides of the SLS TPU/CNT sensing element samples $\left(\Phi 25 \times 3 \mathrm{~mm}^{3}\right)$ were glued with a pair of copper electrodes with conductive silver glue to ensure good contact between the samples and the electrodes. The sensing elements were compressed by jogging, and a high-precision multimeter was used to record changes in their resistance. 
Fig. 2 TPU/CNT parts. a Layerseparation part. b Warping part

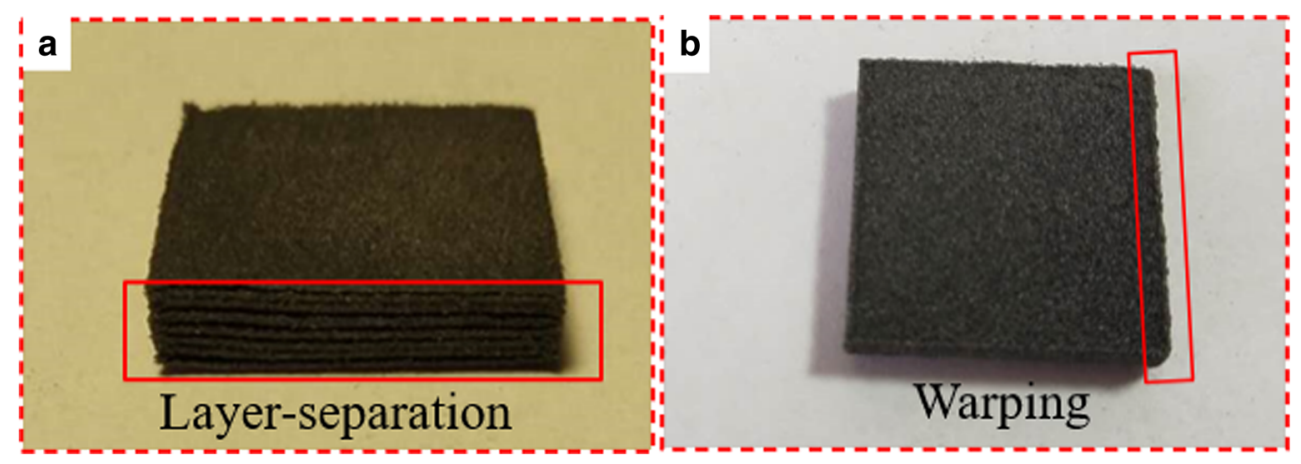

\subsubsection{Piezoresistive sensitivity}

The piezoresistive sensitivity of the TPU/CNT sensing elements can be expressed by the following formula [29]:

$S=\frac{\left(\Delta R / R_{0}\right) \%}{\Delta P}$

$\Delta R=\left|R-R_{0}\right|$

where $\Delta P$ is the relative loaded compressive force, $R_{0}$ is the initial resistance, and $R$ is the resistance. To allow the TPU/ CNT sensor element to have a good resolution under highpressure loading, the sensitivity at $150 \mathrm{kPa}$ was considered to be one of the evaluation indicators of the orthogonal experiment.

\subsubsection{Density}

The mass and dimensions of the TPU/CNT sensing elements were measured with an analytical balance and a vernier caliper. Herein, the density $\rho$ is calculated by Eq. 3:

$\rho=W /(l \cdot b \cdot h)$

where $W$ denotes the mass of sensing elements (g), $l$ represents the length of sensing elements $(\mathrm{mm}), b$ is the width of sensing elements (mm), and $h$ denotes the thickness of sensing elements (mm).

\subsubsection{Dimensional precision}

A rectangular specimen with dimensions of $20 \mathrm{~mm} \times 20 \mathrm{~mm} \times$ $6 \mathrm{~mm}$ was used in the precision analysis. The actual dimensions of the TPU/CNT sensing elements were measured using a vernier caliper. Herein the dimensional precision is calculated via Eq. 4:

$\delta(\%)=\left(1-\frac{L_{0}-L}{L_{0}}\right)$

where $\delta$ represents the dimensional precision of sensing elements $(\%), L_{0}$ denotes the standard dimension of sensing elements ( $\mathrm{mm}$ ), and $L$ indicates the actual dimension of sensing elements $(\mathrm{mm})$. The dimension of TPU/CNT sensing elements in the $Z$ direction (direction of thickness) was also measured, and the $Z$-dimensional precision $\delta_{z}$ was calculated (\%).

\section{Results and discussion}

\subsection{Single-factor analysis}

The piezoresistive sensitivity, Z-dimensional precision, and density of TPU/CNT sensing elements were used to evaluate the effect of SLS processes on the sensing performance and molding quality of TPU/CNT sensing elements, as shown in Table 2. The order of the range value of the piezoresistive sensitivity of TPU/CNT sensing elements from large to small was as follows: laser power $>$ layer thickness $>$ scan spacing. These results illustrated that the laser power had the greatest effect on piezoresistive sensitivity, while scan spacing had the least effect. Layer thickness had the largest effect on density, while laser power had the least influence, and scan spacing had the greatest influence on $Z$-dimensional precision, while laser power had the least influence.
Table 1 Factors and levels of laser sintering test

\begin{tabular}{llll}
\hline Levels & Laser power $(\mathrm{W}),[\mathrm{A}]$ & Scan spacing $(\mathrm{mm}),[\mathrm{B}]$ & Layer thickness $(\mathrm{mm}),[\mathrm{C}]$ \\
\hline 1 & 19 & 0.10 & 0.10 \\
2 & 22 & 0.15 & 0.15 \\
3 & 25 & 0.20 & 0.20 \\
4 & 28 & 0.25 & 0.25 \\
\hline
\end{tabular}


Fig. 3 A schematic diagram of the test setup

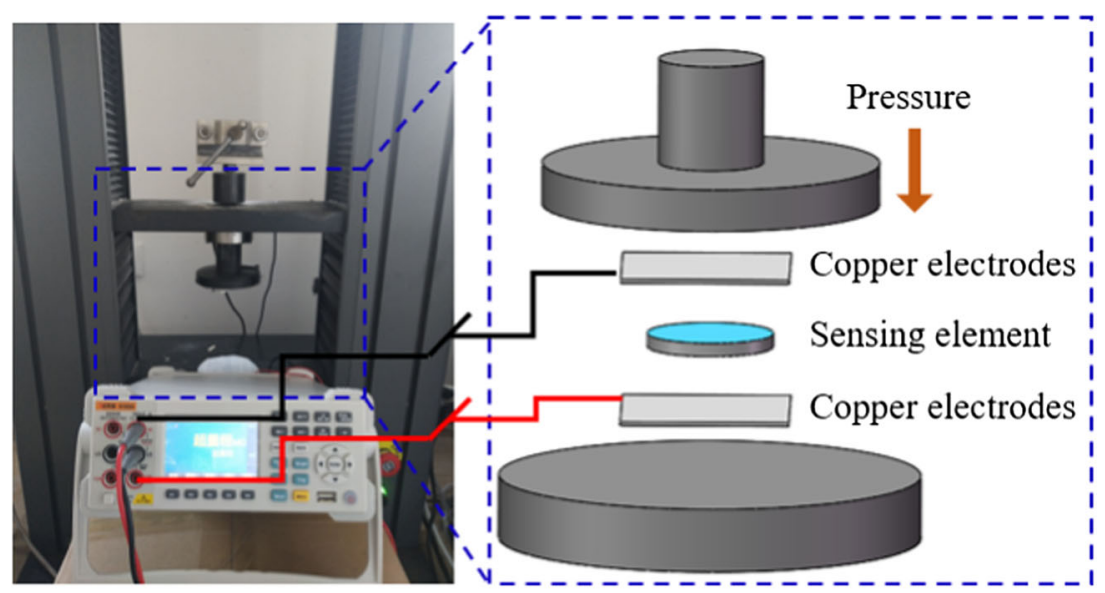

Multimeter(Victor 8165A) Mechanical testing machine (Byes-3003)

\subsubsection{Analysis of the effect of process parameters on piezoresistive sensitivity}

Through the orthogonal experiment, the effects of the SLS process on the piezoresistive sensitivity of TPU/CNT sensing elements were obtained, as shown in Fig. 4. Figure 4a shows that with increasing laser power, the sensitivity of TPU/CNT sensing elements was increased. Owing to low laser power, the internal pores of the TPU/CNT sensing elements were too large, and the distance between the CNTs distributed in the TPU particles was too wide (Fig. 5a, b). It was not easy to form a conductive path. As the pressure increased, the number of conductive paths to be constructed was less than the number to be destroyed, resulting in smaller changes in piezoresistive sensitivity. As the laser power increased, the TPU particles inside the sensing element melted more (Fig. $5 \mathrm{c}$ ), the conductive particle spacing was reduced, and it was easier to form a conductive path during loading. Additionally, it can be seen from Fig. $5 \mathrm{c}$ and $\mathrm{d}$ that when the laser power was $25 \mathrm{~W}$, the inside of the sensing element was already dense, and when the power was increased to $28 \mathrm{~W}$, spherical holes appeared inside the sensing element. This was because higher power causes the materials to overburn, and the generated gas could not be discharged and formed smaller closed holes, which led to a continued increase in sensitivity. Research by scholars such as Chen et al. [10] and Jung et al. [30] has shown that the micropores and porous structure may, to some extent, increase the sensitivity of sensing elements, which also confirms the phenomenon produced in this study.

Figure $4 \mathrm{~b}$ shows that as the scan spacing increased, the sensitivity first decreased and then increased. For TPU/CNT sensing elements with different scan spacings, the difference in internal pores mainly occurred in every single layer of the sensors. Through the transverse deformation of the TPU particles, the pores of the sensing element were reduced and the sensitivity was improved. As shown in Fig. 6a, when the scan spacing was small $(0.10 \mathrm{~mm})$, the internal pore spacing was small, and conductive paths were easily formed under pressure. The pores in the single layer became larger with an increase of the scan spacing $(0.15-0.20 \mathrm{~mm})$, and the distance caused by the increase of the scan spacing was longer, and the damaged conductive path was larger than the constructed conductive path, resulting in a decrease in sensitivity. Since the laser spot diameter is $0.20 \mathrm{~mm}$, when the scanning distance increased to $0.25 \mathrm{~mm}$, the powder inside the TPU/CNT sensing element was not completely fused, and there were fewer conductive paths formed in the lateral direction. As the pressure increased, the TPU particles were deformed laterally, the distance between the conductive particles was shortened, more conductive paths were constructed, and the sensitivity increased.

Figure $4 \mathrm{c}$ shows that as the layer thickness increased, the sensing element sensitivity first increased and then decreased. When the layer thickness was $0.1 \mathrm{~mm}$, the inside of the sensing element was dense (Fig. 6b), and the polyurethane
Table 2 Range values of the effect of process parameters on the quality of TPU/CNT sensing elements

\begin{tabular}{llll}
\hline Levels & Laser power $(\mathrm{W}),[\mathrm{A}]$ & Scan spacing $(\mathrm{mm}),[\mathrm{B}]$ & Layer thickness $(\mathrm{mm}),[\mathrm{C}]$ \\
\hline Sensitivity $(\% / \mathrm{kPa})$ & 5.5 & 1.138 & 5.229 \\
Density $\left(\mathrm{g} / \mathrm{cm}^{3}\right)$ & 0.102 & 0.173 & 0.219 \\
Z-dimensional precision $(\%)$ & 2.188 & 8.646 & 4.438 \\
\hline
\end{tabular}



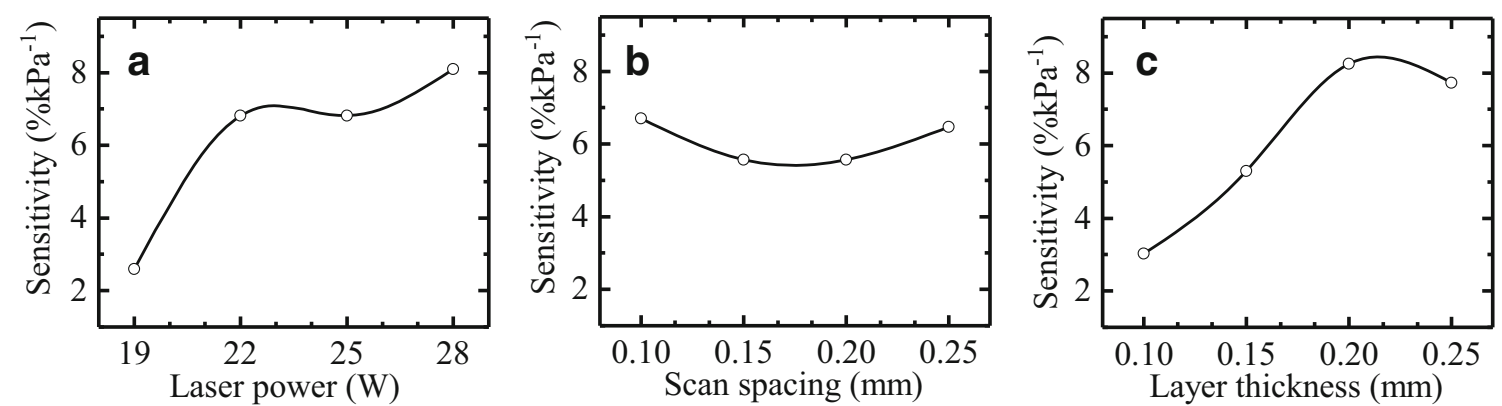

Fig. 4 Effects of process parameters on the piezoresistive sensitivity of TPU/CNT sensing elements. a Laser power. b Scan spacing. $\mathbf{c}$ Layer thickness

particles moved less under applied pressure. The transition was mainly caused by the change of the internal conduction particle spacing, and the resistance change was not obvious, so the sensitivity was small. As the thickness of the layer increased, pores were generated inside (Fig. 6c). The possibility of contact between internal conductive particles gradually increased under applied pressure, thus increasing sensitivity. However, when the layer thickness increased to $0.25 \mathrm{~mm}$, it was difficult to form a conductive path. When pressure was applied, the number of conductive paths constructed was smaller than the number destroyed, resulting in smaller changes in piezoresistive sensitivity.

\subsubsection{Analysis of the effects of process parameters on density}

The effects of the process parameters on the density of TPU/ CNT sensing elements are shown in Fig. 7. Figure 7a shows that with an increase in laser power, the laser energy absorbed by TPU/CNT powder increased, the powder was completely fused, and the quantities of the internal pores of TPU/CNT sensing elements decreased. Thus, the density of TPU/CNT sensing elements increased. When the input power of the laser remained constant, Fig. $7 \mathrm{~b}$ and $\mathrm{c}$ show that with the increase in scan spacing, the re-sintering zone was reduced, the power absorbed by the TPU/CNT powder gradually decreased, the powder was not fully fused, and the amounts of internal pores gradually increased. Thus, the densities of TPU/CNT sensing elements decreased. With increasing layer thickness, the power absorbed by the combination zone between layers decreased, and the number of internal pores of TPU/CNT sensing elements increased. Therefore, the density of sensing elements decreased.

\subsubsection{Analysis of the effect of process parameters on Z-dimensional precision}

The effects of the process parameters on the $Z$-dimensional precision of TPU/CNT sensing elements are shown in Fig. 8. Figure $8 \mathrm{a}-\mathrm{c}$ show that with increased laser power, the power absorbed by TPU/CNT powder increased. The power diffused
Fig 5 Micromorphology of the cross sections of the TPU/CNT sensing element with different laser power values. a Laser power 19 W. b A magnified image of a. c Laser power $25 \mathrm{~W}$. d Laser power $28 \mathrm{~W}$
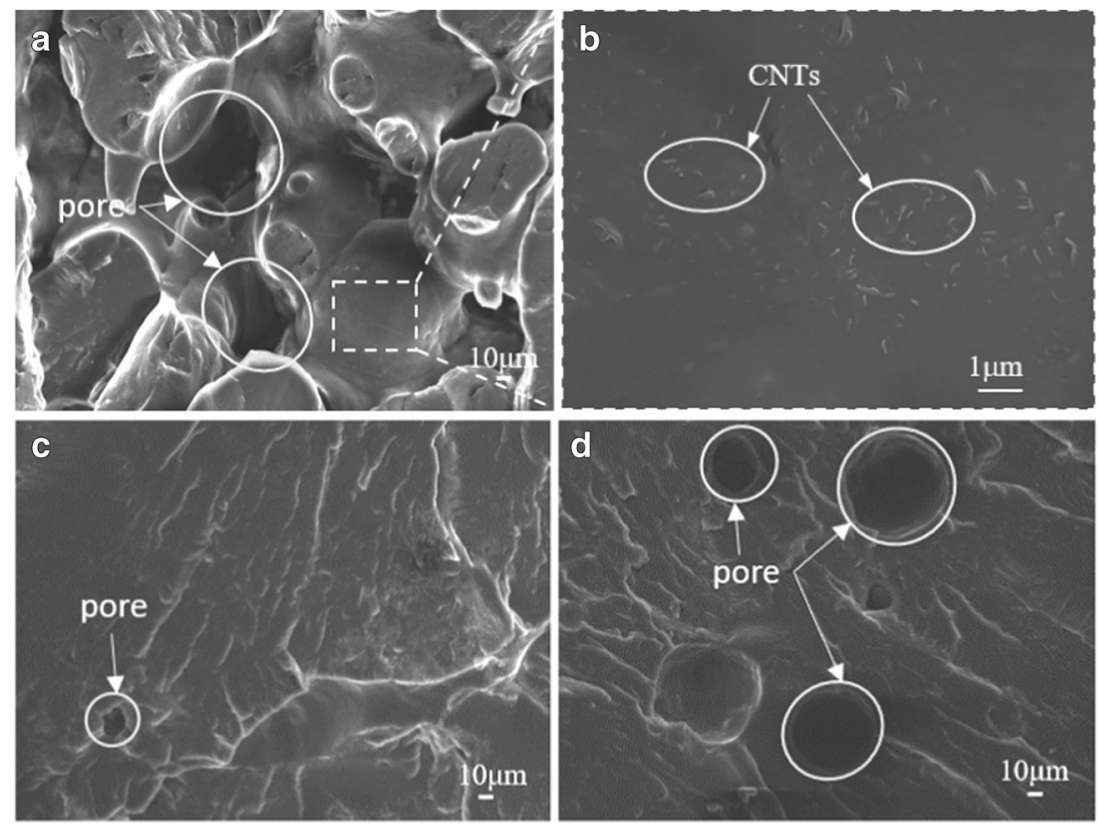
Fig 6 a Schematic diagram of the conductive path formed by TPU/ CNT sensing elements with different scan spacings after compression. b Micromorphology of the cross sections of the TPU/ CNT sensing element with a layer thickness of $0.10 \mathrm{~mm}$. c Micromorphology of the cross sections of the TPU/CNT sensing element with a layer thickness of $0.15 \mathrm{~mm}$
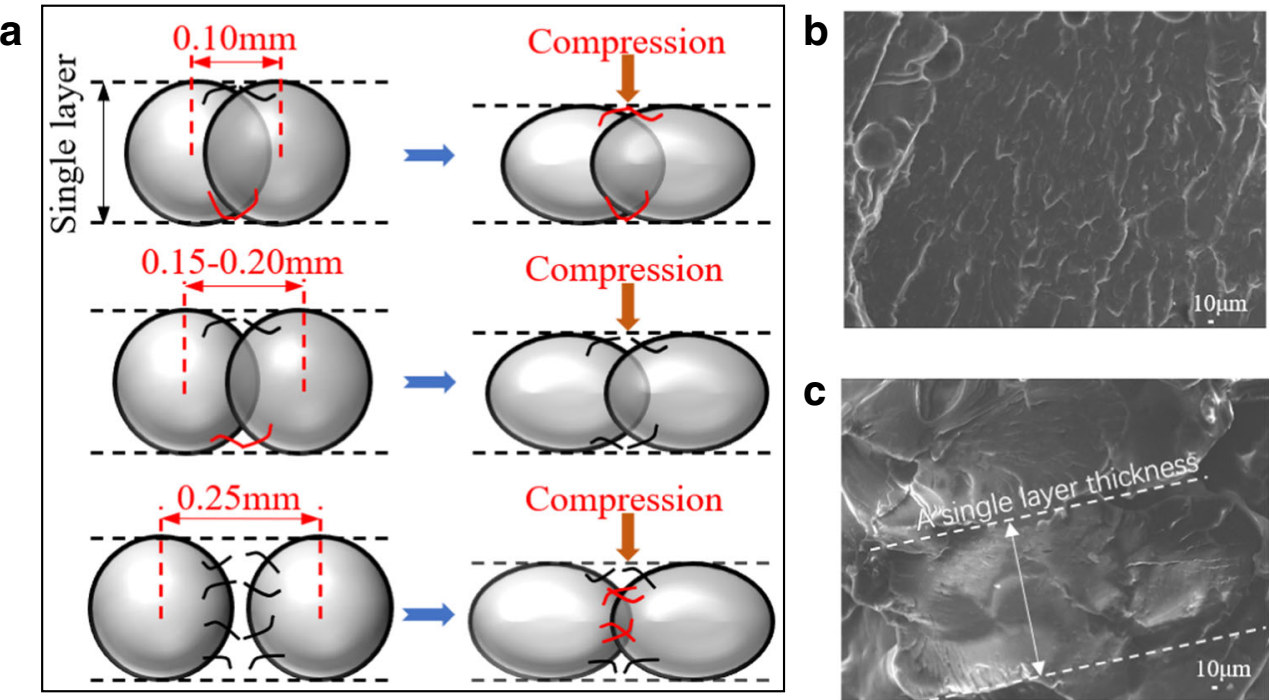

along the laser sintering path and the sintered depth was increased, which increased the dimension of TPU/CNT sensing elements in the $Z$ direction, decreasing the $Z$-dimensional precision. Following an increase in scan spacing, the re-sintering zone was reduced, and the sintered depth decreased, resulting in increased $Z$-dimensional precision. When the input power of the laser remained constant, with increasing layer thickness, the sintered depth decreased, and thus, the Z-dimensional direction of TPU/CNT sensing elements decreased. Therefore, $Z$-dimensional precision improved.

\subsection{Multi-index synthetic analysis}

\subsubsection{Synthetic weighted evaluation}

Multi-index test results were evaluated using a synthetic weighted scoring method. The synthetic weighted scoring method is a method by which multi-index weights were determined based on the importance of each test index in the whole test. The piezoresistive sensitivity, density, and Z-dimensional precision test results were transformed into the single-index test results, and then the test scheme was optimized according to a single-index analysis method.
The piezoresistive sensitivity, density, and Z-dimensional precision were transformed into dimensionless forms. The transformations were performed following Eqs. 5-7:

$$
\begin{aligned}
& Z_{1}=\left(S-S_{\min }\right) /\left(S_{\max }-S_{\min }\right) \\
& Z_{2}=\left(\rho-\rho_{\min }\right) / \rho_{\max }-\rho_{\min } \\
& Z_{3}=\left(\delta-\delta_{\min }\right) /\left(\delta_{\max }-\delta_{\min }\right)
\end{aligned}
$$

\subsubsection{Synthetic weights of test index}

Sensitivity affects the sensing performance and measurement range of the pressure-sensing element. When the measurement range is sufficient, the higher the sensitivity, the better the resolution of pressure detection. The density of TPU/CNT sensing elements was higher, indicating that the inner structure of the sensing element was denser and that the internal conductive network structure was not easily destroyed. The higher $Z$-dimensional precision revealed better forming precision. The test indexes were given weights based on the importance of the test indexes. In general, higher weights were given to indexes of more subjective importance.
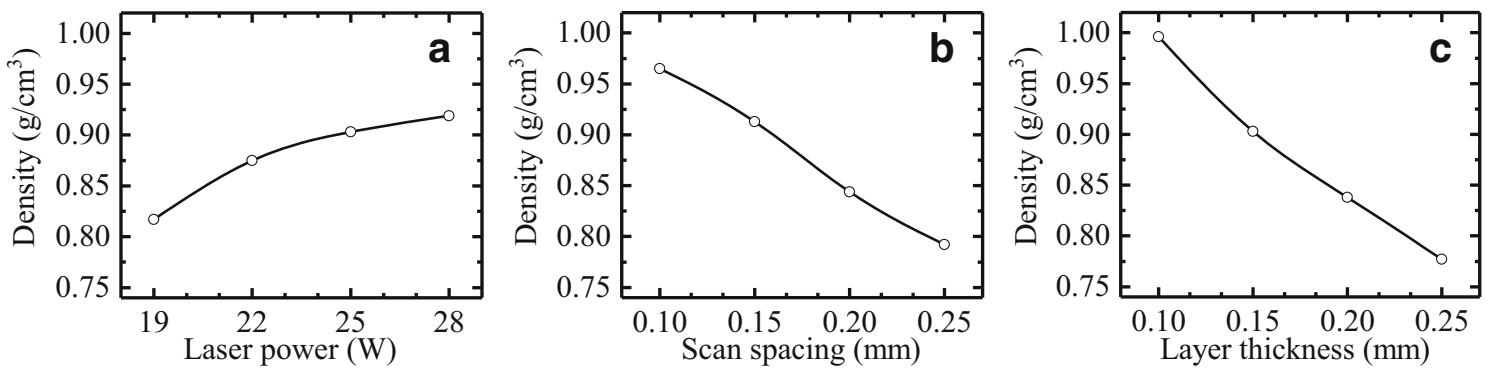

Fig 7 Effect of process parameters on the density of TPU/CNT sensing elements. a Laser power. b Scan spacing. c Layer thickness 

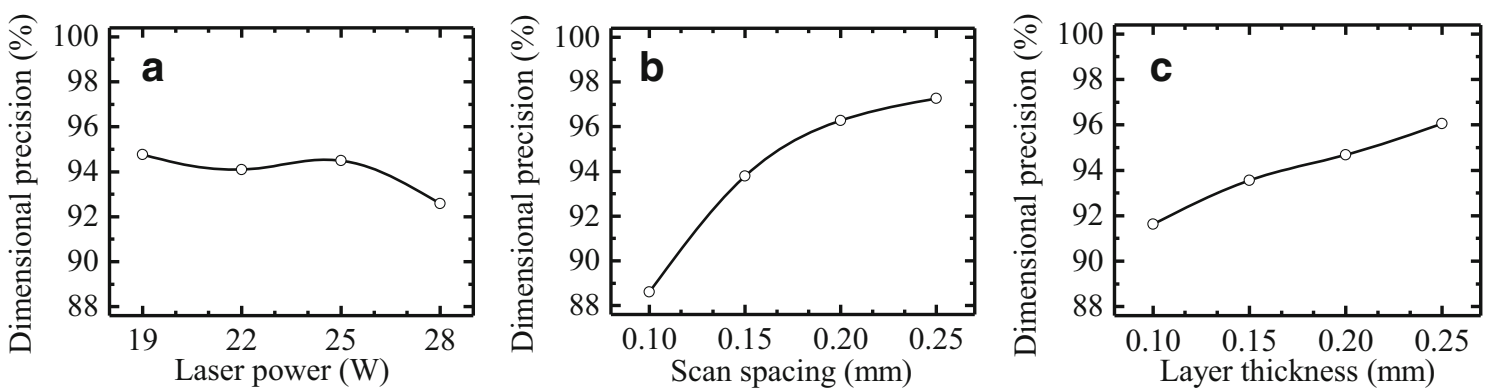

Fig. 8 Effect of process parameters on Z-dimensional precision of TPU/CNT sensing elements. a Laser power. b Scan spacing. $\mathbf{c}$ Layer thickness

The effect of piezoresistive sensitivity was the key consideration, and the density and $Z$-dimensional precision were secondary considerations. The weights of piezoresistive sensitivity, density, and Z-dimensional precision were $\lambda_{1}=0.5$, $\lambda_{2}=0.3$, and $\lambda_{3}=0.2$, respectively. Therefore, the synthetic weighted scoring values are shown by Eq. 8:

$Z=\lambda_{1} Z_{1}+\lambda_{2} Z_{2}+\lambda_{3} Z_{3}$

\subsubsection{Results and analysis of the test}

Test data were processed using the synthetic weighted scoring method. The range analysis results of piezoresistive sensitivity, density, and Z-dimensional precision were obtained, as shown in Table 3.

Scan spacing and layer thickness were large because the laser power of the fourth group of orthogonal experiments was too small, resulting in a slight layering phenomenon in the laser sintering sensing elements. Hence, so the resistance signal could not be detected, and the sensitivity was set to 0 . Table 3 shows that the range of laser power (A) was the largest, which had the greatest effect on the test index. Level 4 was suitable for A. The range of layer thickness (C) was smaller than that of A, implying its effect was less. Level 3 was suitable for $\mathrm{C}$. The range of scan spacing (B) was the smallest; hence, its influence was the least. Level 2 was suitable for $\mathrm{C}$.

Table 3 Laser sintering process scheme and experimental results of TPU/CNT sensing elements

\begin{tabular}{|c|c|c|c|c|c|c|c|}
\hline \multirow{2}{*}{$\begin{array}{l}\text { Serial } \\
\text { number }\end{array}$} & \multirow{2}{*}{$\begin{array}{l}\text { Laser power }(\mathrm{W}) \\
{[\mathrm{A}]}\end{array}$} & \multirow{2}{*}{$\begin{array}{l}\text { Scan spacing }(\mathrm{mm}) \\
{[\mathrm{B}]}\end{array}$} & \multirow{2}{*}{$\begin{array}{l}\text { Layer thickness (mm) } \\
\text { [C] }\end{array}$} & \multicolumn{4}{|l|}{ Test index } \\
\hline & & & & $\begin{array}{l}\text { Sensitivity } \\
(\% / \mathrm{kPa})\end{array}$ & $\begin{array}{l}\text { Density } \\
\left(\mathrm{g} / \mathrm{cm}^{3}\right)\end{array}$ & $\begin{array}{l}\text { Dimensional precision } \\
(\%)\end{array}$ & $\begin{array}{l}\text { Synthetic } \\
\text { weight }\end{array}$ \\
\hline 1 & 19 & 0.1 & 0.1 & 2.378 & 1.018 & 86.33 & 0.353 \\
\hline 2 & 19 & 0.15 & 0.15 & 5.192 & 0.893 & 94.94 & 0.482 \\
\hline 3 & 19 & 0.2 & 0.2 & 2.811 & 0.725 & 97.94 & 0.330 \\
\hline 4 & 19 & 0.25 & 0.25 & 0 & 0.631 & 99.83 & 0.200 \\
\hline 5 & 22 & 0.1 & 0.15 & 5.405 & 0.989 & 87.89 & 0.454 \\
\hline 6 & 22 & 0.15 & 0.1 & 8 & 0.875 & 94.56 & 0.554 \\
\hline 7 & 22 & 0.2 & 0.25 & 11.243 & 0.733 & 97.92 & 0.607 \\
\hline 8 & 22 & 0.25 & 0.2 & 2.605 & 0.902 & 96.00 & 0.421 \\
\hline 9 & 25 & 0.1 & 0.2 & 6.706 & 0.941 & 90.00 & 0.492 \\
\hline 10 & 25 & 0.15 & 0.25 & 7.351 & 0.834 & 96.28 & 0.529 \\
\hline 11 & 25 & 0.2 & 0.1 & 5.4 & 1.012 & 94.78 & 0.572 \\
\hline 12 & 25 & 0.25 & 0.15 & 7.783 & 0.824 & 96.94 & 0.546 \\
\hline 13 & 28 & 0.1 & 0.25 & 12.325 & 0.910 & 90.22 & 0.654 \\
\hline 14 & 28 & 0.15 & 0.1 & 1.73 & 1.050 & 89.39 & 0.401 \\
\hline 15 & 28 & 0.2 & 0.15 & 2.815 & 0.904 & 94.44 & 0.406 \\
\hline 16 & 28 & 0.25 & 0.2 & 15.51 & 0.812 & 96.25 & 0.776 \\
\hline K1 & 0.341 & 0.488 & 0.437 & \multicolumn{4}{|c|}{ Optimum combination: A4C3B2 } \\
\hline K2 & 0.509 & 0.492 & 0.472 & & & & \\
\hline K3 & 0.535 & 0.479 & 0.538 & & & & \\
\hline K4 & 0.559 & 0.486 & 0.498 & & & & \\
\hline $\mathrm{R}$ & 0.218 & 0.013 & 0.101 & & & & \\
\hline
\end{tabular}


Table 4 Contrast data of test results

\begin{tabular}{|c|c|c|c|c|c|c|}
\hline \multirow{2}{*}{$\begin{array}{l}\text { Test } \\
\text { contrast }\end{array}$} & \multicolumn{2}{|c|}{ Low loading } & \multicolumn{2}{|c|}{ High loading } & \multirow{2}{*}{$\begin{array}{l}\text { Density } \\
\left(\mathrm{g} / \mathrm{cm}^{3}\right)\end{array}$} & \multirow{2}{*}{$\begin{array}{l}Z- \\
\text { dimensiona } \\
\text { precision } \\
(\%)\end{array}$} \\
\hline & $\begin{array}{l}\text { Pressure } \\
(\mathrm{kPa})\end{array}$ & $\begin{array}{l}\text { Sensitivity } \\
(/ \mathrm{kPa})\end{array}$ & $\begin{array}{l}\text { Pressure } \\
(\mathrm{kPa})\end{array}$ & $\begin{array}{l}\text { Sensitivity } \\
(/ \mathrm{kPa})\end{array}$ & & \\
\hline Optimized & $3-70$ & 1.357 & $70-240$ & 0.0328 & 0.916 & 94.38 \\
\hline Optimum & $30-70$ & 0.991 & $70-240$ & 0.162 & 0.812 & 96.25 \\
\hline
\end{tabular}

Thus, A4C3B2 was the optimum combination of various factors, namely, the laser power was $28 \mathrm{~W}$, the scan spacing was $0.15 \mathrm{~mm}$, and the layer thickness was $0.2 \mathrm{~mm}$.

The optimized process parameters were compared with the optimum combination of comprehensive weighted scores (Table 3), and the analysis results are presented in Table 4. Table 4 shows that using the optimized SLS process parameters, the density of the TPU/CNT sensing elements was $12.8 \%$ higher than that of the optimum combination, and the Z-dimensional precision was reduced by $1.94 \%$. To further understand the changes of the optimum process parameters and the optimized sensing performance of the TPU/CNT sensing elements, the piezoresistive sensitivity of the TPU/CNT sensing elements are plotted in Fig. 9a for comparison. Figure 9a shows that when high loading was applied, although the TPU/CNT sensing elements prepared with the optimum process parameters were more sensitive than the optimized sensing element, they were more sensitive to detecting large pressure loads. However, due to large internal pores in the TPU/ CNT sensors, no internal conductive network was formed in the initial stage of pressure loading, and the resistance signal could not be detected until the load reached $30 \mathrm{kPa}$. Additionally, during the pressure unloading process, the optimum parameter sensing elements had a certain hysteresis, and when unloaded to $40 \mathrm{kPa}$, the resistance could not be detected when unloaded to $40 \mathrm{kPa}$. It may be because there were many pores in the TPU/CNT sensing elements. When high loading is applied, the internal conductive network structure was destroyed and could not be restored to its initial resistance.

A wide pressure detection range $(3-240 \mathrm{kPa})$ and no obvious hysteresis during pressure loading and unloading were seen because the optimized TPU/CNT sensing elements could detect small pressures. Although sensitivity was low under large pressure loads, it was almost the same as that of the flexible pressure-sensing elements reported by He et al. [31] and Yang et al. [32]; thus, the optimized TPU/CNT sensing elements were used for subsequent research on their sensing performance.

In addition to sensitivity and pressure detection range, the response time and reproductivity during cyclic testing were also important indicators for identifying important sensors. Therefore, we conducted a follow-up experimental test on
Fig. 9 a Comparison of resistance change $\left(\Delta R / R_{0}\right)$ curves of the optimized TPU/CNT sensing elements and optimum TPU/ CNT sensing elements. b The resistance changes after a sudden increase in pressure from 22 to 30 $\mathrm{kPa}$. c Long-term stability of the TPU/CNT sensing elements over 500 cycles at a compression strain of $10 \%$
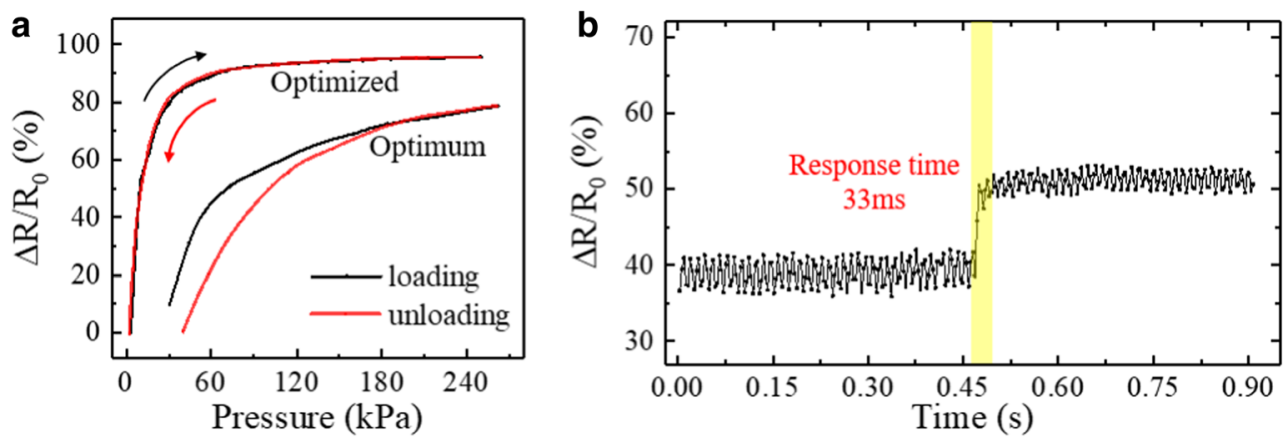

C

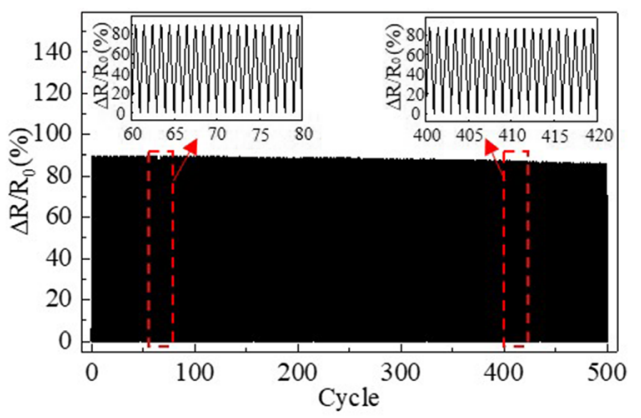


Table 5 Contrast data of test results

\begin{tabular}{llllll}
\hline Materials & Sensitivity: /kPa & Pressure range: $\mathrm{kPa}$ & $\begin{array}{l}\text { Response } \\
\text { time: } \mathrm{ms}\end{array}$ & Processing technology & Ref. number \\
\hline TPU/CNT composites & $0.162-0.991$ & $3-240$ & 33 & SLS technology & This manuscript \\
MWCNT and TPU foam & $0.013-0.032$ & $0-200$ & 29 & Direct attachment method & {$[15]$} \\
MWCNT/PU composites & 0.0428 & $0-63$ & - & Casting technology & [31] \\
Graphene foam & 0.36 & $0-5$ & 80 & Chemical and thermal reduction processes & {$[32]$} \\
MWCNT and rGO loaded PU foam & $0.034-0.088$ & $0-48.8$ & 30 & Drying directly after soaking & {$[33]$} \\
\hline
\end{tabular}

the sensing performance of the optimized TPU/CNT sensing elements. As shown in Fig. 9b, the response time to a sudden pressure input of $30 \mathrm{kPa}$ was $33 \mathrm{~ms}$. Compared with the response times of other sensors, this response time was almost identical to the value from other sensors [15, 33]. Meanwhile, the durability and stability of the TPU/CNT sensing elements were further evaluated over 500 cycles at a compression strain of $10 \%$ (Fig. 9c). The resistance changed periodically, and no obvious fluctuation of the sensing signal was observed. After 500 compression cycles, the strain sensing pattern remained stable, and the repeatability error was less than $3.58 \%$, indicating that the conductive network was very stable, which was beneficial for stable sensing. All of these indicate that the optimized TPU/CNT sensing elements have high repeatability, fast response, stability, and durability. To compare the optimized TPU/CNT sensing elements with flexible pressure sensors prepared by other processing technologies, the comparison report of the main parameters of the latest pressure sensor is presented in Table 5. The report shows that the SLS TPU/CNT sensor has the advantages of high sensitivity, good stability, and fast response time, but with a small pressure, it has no resistance response. In a future work, we will continue to explore post-processing and broaden the pressure measurement range.

\subsection{Application}

To illustrate the applicability of the sensing performance of TPU/CNT sensing elements, we used a three-dimensional scanner to scan and model the human foot and used laser-sintered TPU/CNT composites to prepare pressure-sensing insoles to detect the distribution of plantar pressure (Fig. 10a). The upper

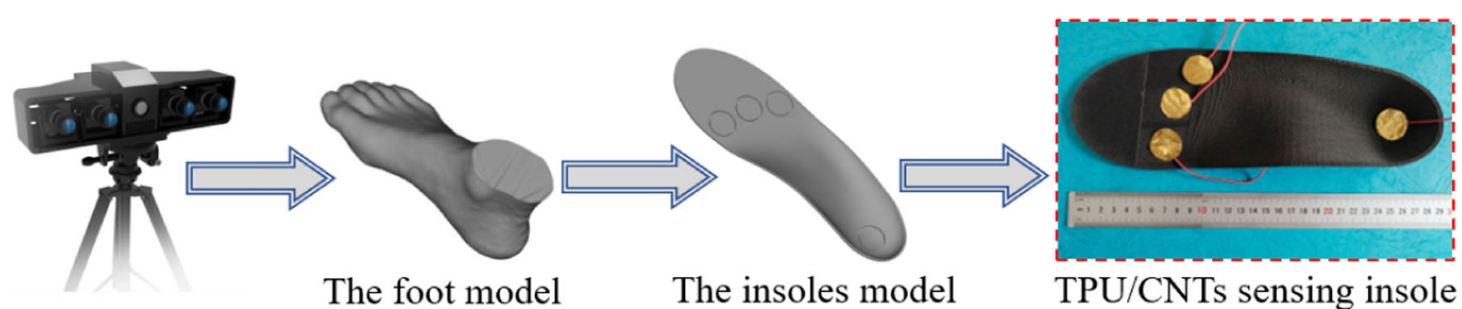

a

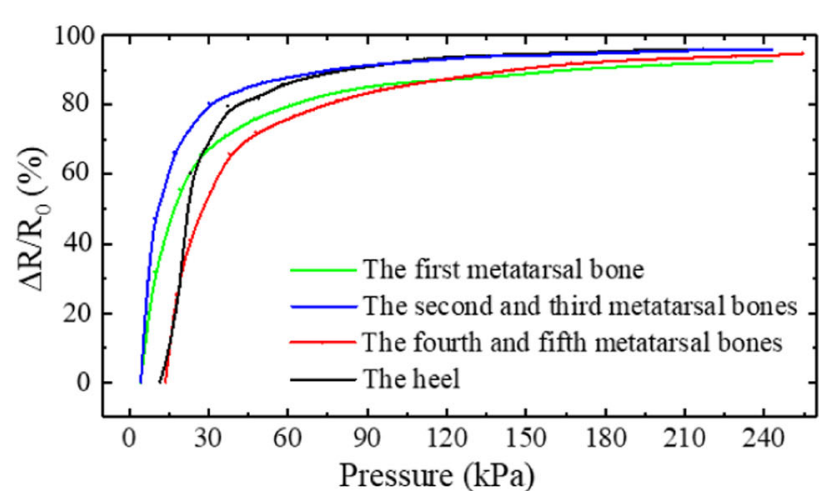

b

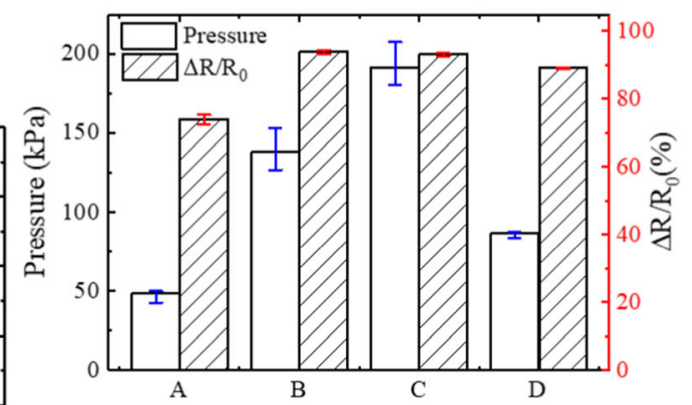

A-The first metatarsal bone B-The second and third metatarsal bones C- - The fourth and fifth metatarsal bones D-The heel

Fig. 10 a The TPU/CNT sensing insole preparation process. b Resistance change $\left(\Delta R / R_{0}\right)$ of four sensing positions under single-point pressure-loading experiment. $\mathbf{c}$ Fitting values of pressure distribution and resistance changes at four sensing positions of the sole 
and lower sides of the four main positions of the sensing insole were, respectively, bonded with a pair of copper electrodes and conductive silver glue to ensure good contact between the insole and the electrodes. The four groups of electrodes were connected to a high-precision multimeter to collect the resistance signal of the plantar pressure. The four pressure-sensing positions were the first metatarsal bone, the second and third metatarsal bones, the fourth and fifth metatarsal bones, and the heel (Fig. 10a).

Before the test, the pressure-sensing insole was placed on the pressure-loading platform, and the single-point pressureloading experiment was carried out on the four test points. To calibrate the pressure load of each test point, control the change of the loading force through the controller and record the resistance change of each sensing position. During the test, the subject took off his shoes, with feet shoulder-width apart and stood on the sensing insole. The test time was 9-10 s, and the test was performed five times, and the results were inputted into a computer; the data were fitted to measure the pressure experienced by each test point.

Figure $9 \mathrm{~b}$ shows the resistance changes of the four pressure detection positions. Since the sensing insoles were modeled according to the human foot, the generated complex threedimensional curved surface had a slight impact on the resistance changes of each test point (Fig. 10b). Figure 10c shows the fitting values of pressure distribution and resistance changes at four sensing positions of the insole. The highest pressure was detected at the fourth and fifth metatarsal bones, at approximately $191.22 \mathrm{kPa}$, followed by the second and third metatarsal bones and the heel, and the first metatarsal bone experienced the least stress. The detected pressure range was consistent with the normal adult plantar pressure range reported by Zhang [34] and Veves et al. [35]. Therefore, it has been demonstrated that the optimized TPU/CNT sensing elements can be used to measure biological and motion signals, and sensing elements can be installed according to demand, providing new technical solutions for human movement detection, smart wearable devices, medical rehabilitation, and other fields.

\section{Conclusions}

The orthogonal experiment design method and the comprehensive weight method were used to optimize the multiobjectives and prepare the TPU/CNT pressure sensor element with good flexible sensing performance. The optimized SLS TPU/CNT sensing element density was increased by $12.8 \%$; the $Z$-direction accuracy was reduced by $1.94 \%$, and the sensing element has a wide pressure detection range (3-240 $\mathrm{kPa})$ and high sensitivity $(1.357 / \mathrm{kPa}$ for $3-70 \mathrm{kPa}, 0.0328 / \mathrm{kPa}$ for $70-240 \mathrm{kPa}$ ); its response time can reach $33 \mathrm{~ms}$, and it still has good stability and durability after 500 loads (10\% strain).
Finally, the flexible pressure-sensing element was successfully applied to detect human foot pressure. This work provides a good research foundation for smart wearable devices and human motion detection and presents a simple and effective method to develop flexible pressure sensors.

Author contributions Methodology and data curation, Y.Z. and K.J.; formal analysis, Y.Y.; design and performance of the experiments, S.G. and H.Z.; writing — original draft preparation, Y.Z.; writing — review and editing, J.L.; project administration, Y.G. and J.L.; funding acquisition and resources, Y.G. All authors have read and agreed to the published version of the manuscript.

Funding This research was funded by National Natural Science Foundation of China, grant number 52075090; the key projects of provincial fund, grant number ZD2017009; Fundamental Research Funds for the Central Universities, grant number 2572019AB20; the Key National Research and Development Programs, grant number 2017YFD0601004; and Double First-Class Fund of Northeast Forestry University, grant number 41113253.

\section{Compliance with ethical standards}

Conflict of interest The authors declare that they have no conflict of interest.

Open Access This article is licensed under a Creative Commons Attribution 4.0 International License, which permits use, sharing, adaptation, distribution and reproduction in any medium or format, as long as you give appropriate credit to the original author(s) and the source, provide a link to the Creative Commons licence, and indicate if changes were made. The images or other third party material in this article are included in the article's Creative Commons licence, unless indicated otherwise in a credit line to the material. If material is not included in the article's Creative Commons licence and your intended use is not permitted by statutory regulation or exceeds the permitted use, you will need to obtain permission directly from the copyright holder. To view a copy of this licence, visit http://creativecommons.org/licenses/by/4.0/.

\section{References}

1. Hu W, Lum GZ, Mastrangeli M, Sitti M (2018) Small-scale softbodied robot with multimodal locomotion. Nature 554:81-85. https://doi.org/10.1038/nature25443

2. Lipomi DJ, Vosgueritchian M, Tee BC, Hellstrom SL, Lee JA, Fox $\mathrm{CH}$, Bao Z (2011) Skin-like pressure and strain sensors based on transparent elastic films of carbon nanotubes. Nat Nanotechnol 6: 788-792. https://doi.org/10.1038/nnano.2011.184

3. Wang Z, Hao Z, Yu S, Huang C, Pan Y, Zhao X (2020) A wearable and deformable graphene-based affinity nanosensor for monitoring of cytokines in biofluids. Nanomaterials 10:1503-1512. https://doi. org/10.3390/nano10081503

4. Zhuang Y, Guo Y, Li J, Jiang K, Yu Y, Zhang H, Liu D (2020) Preparation and laser sintering of a thermoplastic polyurethane carbon nanotube composite-based pressure sensor. RSC Adv 10: 23644-23652. https://doi.org/10.1039/D0RA04479B

5. Lu H, Zhang M, Yang Y, Huang Q, Fukuda T, Wang Z, Shen Y (2018) A bioinspired multilegged soft millirobot that functions in both dry and wet conditions. Nat Commun 9:3944-3952. https:// doi.org/10.1038/s41467-018-06491-9 
6. Liu H, Li Q, Zhang S, Yin R, Liu X, He Y, Dai K, Shan C, Guo J, Liu C, Shen C, Wang X, Wang N, Wang Z, Wei R, Guo Z (2018) Electrically conductive polymer composites for smart flexible strain sensors: a critical review. J Mater Chem C 6:12121-12141. https:// doi.org/10.1039/C8TC04079F

7. Liu H, Huang W, Yang X, Dai K, Zheng G, Liu C, Shen C, Yan X, Guo J, Guo Z (2016) Organic vapor sensing behaviors of conductive thermoplastic polyurethane-graphene nanocomposites. J Mater Chem 4:4459-4469. https://doi.org/10.1039/C6TC00987E

8. Yin R, Yang S, Li Q, Zhang S, Shen C (2020) Flexible conductive $\mathrm{Ag}$ nanowire/cellulose nanofibril hybrid nanopaper for strain and temperature sensing applications. Sci Bull 65:899-908. https://doi. org/10.1016/j.scib.2020.02.020

9. Liu H, Li Q, Bu Y, Zhang N, Shen C (2019) Stretchable conductive nonwoven fabrics with self-cleaning capability for tunable wearable strain sensor. Nano Energy 2019:104143-104152. https://doi.org/ 10.1016/j.nanoen.2019.104143

10. Chen XY, Liu H, Zheng YJ, Zhai Y, Liu X, Liu C, Mi L, Guo Z, Shen C (2019) Highly compressible and robust polyimide/carbon nanotube composite aerogel for high-performance wearable pressure sensor. ACS Appl Mater Interfaces 11:42594-42606. https:// doi.org/10.1021/acsami.9b14688

11. Small WR, Panhuis MIH (2007) Inkjet printing of transparent, electrically conducting single-waited carbon-nanotube composites. Small 3:1500-1503. https://doi.org/10.1002/smll.200700110

12. Pargoletti E, Cappelletti G (2020) Breakthroughs in the design of novel carbon-based metal oxides nanocomposites for VOCs gas sensing. Nanomaterials 10:1485. https://doi.org/10.3390/ nano10081485

13. Michelis F, Bodelot L, Bonnassieux Y, Lebental B (2015) Highly reproducible, hysteresis-free, flexible strain sensors by inkjet printing of carbon nanotubes. Carbon 95:1020-1026. https://doi.org/10. 1016/j.carbon.2015.08.103

14. Liu H, Huang W, Gao J, Dai K, Zheng G, Liu C, Shen C, Yan X, Guo J, Guo Z (2016) Piezoresistive behavior of porous carbon nanotube-thermoplastic polyurethane conductive nanocomposites with ultrahigh compressibility. Appl Phys Lett 108:011904. https://doi.org/10.1063/1.4939265

15. Lee J, Kim J, Shin Y, Jung I (2019) Ultra-robust wide-range pressure sensor with fast response based on polyurethane foam doubly coated with conformal silicone rubber and CNT/TPU nanocomposites islands. Compos Part B 177:107364.1-107364.10. https://doi. org/10.1016/j.compositesb.2019.107364

16. Zhai Y, Lados DA, LaGoy JL (2014) Additive manufacturing: making imagination the major limitation. JOM 66:808-816. https://doi.org/10.1007/s11837-014-0886-2

17. Ling Z, Wu J, Wang X, Li XF, Zheng JJ (2019) Experimental study on the variance of mechanical properties of polyamide 6 during multi-layer sintering process in selective laser sintering. Int J Adv Manuf Technol 101:1227-1234. https://doi.org/10.1007/s00170018-3004-8

18. Kucewicz M, Baranowski P, Malachowski J, Poplawski A, Platek P (2018) Modelling, and characterization of 3D printed cellular structures. Mater Des 142:177-189. https://doi.org/10.1016/j.matdes. 2018.01.028

19. Abbott AC, Tandon GP, Bradford RL, Koerner H, Baur JW (2018) Process-structure-property effects on ABS bond strength in fused filament fabrication. Addit Manuf 19:29-38. https://doi.org/10. 1016/j.addma.2017.11.002

20. Mohsenizadeh M, Gasbarri F, Munther M, Beheshti A, Davami K (2018) Additively-manufactured lightweight Metamaterials for energy absorption. Mater Des 139:521-530. https://doi.org/10.1016/j. matdes.2017.11.037
21. Yu Y, Guo Y, Jiang T, Jiang K, Li J, Guo S (2017) Laser sintering and post-processing of a walnut shell/Co-PES composite. RSC Adv 7:23176-23181. https://doi.org/10.1039/C7RA00775B

22. Fayed EM, Elmesalamy AS, Sobih M, Elshaer Y (2018) Characterization of direct selective laser sintering of alumina. Int J Adv Manuf Technol 94:2333-2341. https://doi.org/10.1007/ s00170-017-0981-y

23. Li Y, Luo SD, Yang MC, Liang R, Zeng CC (2016) Poisson ratio and piezoresistive sensing: a new route to high-performance $3 \mathrm{D}$ flexible and stretchable sensors of multimodal sensing capability. Adv Funct Mater 26:2900-2908. https://doi.org/10.1002/adfm. 201505070

24. Shirazi SFS, Gharehkhani S, Mehrali M, Yarmand H, Metselaar HSC, Adib Kadri N, Osman NAA (2015) A review on powderbased additive manufacturing for tissue engineering: selective laser sintering and inkjet 3D printing. Sci Technol Adv Mater 16: 033502. https://doi.org/10.1088/1468-6996/16/3/033502

25. Dizon JRC, Espera AH, Chen Q, Advincula RC (2018) Mechanical characterization of 3D-printed polymers. Addit Manuf 20:44-67. https://doi.org/10.1016/j.addma.2017.12.002

26. Flores I, Kretzschmar N, Azman AH, Chekurov S, Pedersen DB, Chaudhuri A (2020) Implications of lattice structures on economics and productivity of metal powder bed fusion. Addit Manuf 31: 100947. https://doi.org/10.1016/j.addma.2019.100947

27. Arısoy YM, Criales LE, Özel T, Lane B, Moylan S, Donmez A (2017) Influence of scan strategy and process parameters on microstructure and its optimization in additively manufactured nickel alloy 625 via laser powder bed fusion. Int J Adv Manuf Technol 90:1393-1417. https://doi.org/10.1007/s00170-016-9429-z

28. Luo RP (2013) Research on synthesis and performance of carbon nanotubes/ graphene/ epoxy resin composites, Master Dissertation, East China University of Science and Technology, Shanghai, China.

29. He Y, Li W, Yang G, Liu H, Lu J, Zheng T, Li X (2017) A Novel method for fabricating wearable, piezoresistive, and pressure sensors based on modified-graphite/polyurethane composite films. Materials (Basel) 10:684-699. https://doi.org/10.3390/ ma10070684

30. Jung S, Kim JH, Kim J, Choi S, Lee J, Park I, Hyeon T, Kim D (2014) Reverse-micelle-induced porous pressure-sensitive rubber for wearable human-machine interfaces. Adv Mater 26:48254830. https://doi.org/10.1002/adma.201401364

31. He Y, Ming Y, Li W, Li Y, Wu M, Song J, Li X, Liu H (2018) Highly stable and flexible pressure sensors with modified multiwalled carbon nanotube/polymer composites for human monitoring. Sensors (Basel) 18:1338. https://doi.org/10.3390/s18051338

32. Yang J, Ye Y, Li X, Lu X, Chen R (2018) Flexible, conductive, and highly pressure-sensitive graphene-polyimide foam for pressure sensor application. Compos Sci Technol 164:187-194. https://doi. org/10.1016/j.compscitech.2018.05.044

33. Tewari A, Gandla S, Bohm S, McNeill CR, Gupta D (2018) Highly exfoliated MWNT-rGO ink wrapped polyurethane foam for piezoresistive pressure sensor applications. ACS Appl Mater Interfaces 10:5185-5195. https://doi.org/10.1021/acsami.7b15252

34. Zhang SL (2006) The investigation of plantar pressure parameters and its distribution, and influencing factors in normal Chinese. Master Dissertation, Sun Yat-sen University, Guangdong, China.

35. Veves A, Fernando DJS, Walewski P, Boulton AJM (1991) A study of plantar pressures in a diabetic clinic population. Foot 1: 89-92. https://doi.org/10.1016/0958-2592(91)90036-B

Publisher's note Springer Nature remains neutral with regard to jurisdictional claims in published maps and institutional affiliations. 